\title{
Rot1, an essential yeast protein, is degraded through the ER-associated protein degradation system (ERAD)
}

\author{
M. Angeles Juanes ${ }^{1,2}$, Carlos A. Martínez-Garay ${ }^{1}$ and M. Carmen Bañóón \\ ${ }^{1}$ Departament de Bioquímica i Biologia Molecular and Estructura de Recerca Interdisciplinar en \\ Biotecnologia i Biomedicina (ERI BIOTECMED), Universitat de València, Burjassot, Valencia, \\ 46100, Spain \\ ${ }^{2}$ Current Address: Department of Biology, Rosenstiel Basic Medical Science Research Center, \\ Brandeis University, Waltham, MA, 02454, USA \\ \Corresponding Authors: juanes@brandeis.edu/majuaor@uv.es; bano@uv.es
}

\begin{abstract}
S. cerevisiae ROT1 is an essential gene which has been related to cell wall biosynthesis, the actin cytoskeleton and protein folding. Rot1 protein is primarily located at the endoplasmic reticulumnuclear membrane facing the lumen where it is translocated through two internal topogenic elements by an SRP-independent posttranslational mechanism which depends on Sec62 to then be $N$-glycosylated at the lumen of the ER. Despite Rot1 protein levels are critical for yeast survival, proper cell cycle progression and morphogenesis, Rot1 protein levels has not been investigated so far. Here we carry out an in vivo study to analyse the Rot1 protein levels and show that Rot1 is a short-lived protein and its turnover is mediated by the ubiquitin proteasome system (UPS), dependent on the main degradation pathway located at the ER, the ER-associated degradation system (ERAD). This finding correlates with genetic interactions we previously found and that suggested a link between Rot1 and the ubiquitin-proteasome system. In addition, by using mutant cells components of the ERAD pathway, we demonstrated that Rot 1 is degraded through the ubiquitin conjugating enzymes (E2) components of ERAD, Ubc6 and Ubc7, and it seems to require only one of the E3 ubiquitin ligases involved in ERAD, Hrd1 but not Doa1.
\end{abstract}

Keywords: Rot1, yeast, ER, degradation, ubiquitin ligases, proteasome

\section{Introduction}

Protein levels in cells are governed not only by protein synthesis but also by protein degradation and is critical for maintaining cellular homeostasis. The ubiquitinproteasome system (UPS) constitutes the major mechanism by which cells acutely alter levels of cytosolic, nuclear, and endoplasmic reticulum (ER) proteins in a highly regulated manner [1, 2]. This occurs generally, but not exclusively, by conjugation with chains of ubiquitin linked through lysine 48 (K48) of ubiquitin, which targets modified proteins to the 26S proteasome (composed by $19 \mathrm{~S}$ and $20 \mathrm{~S}$ particles) for degradation $[3,4]$. It is worth mentioning that the $20 \mathrm{~S}$ particle of eukaryotic proteasomes contains several catalytic subunits that hydrolyze peptide bonds with distinct site specificities. The Pre1 subunit is responsible, at least in part, for the chymotryptic activity of the proteasome [5]. Mutations in Pre1 alone, or in combination with mutations in two other proteasomal subunits, Pre2 or Pre4, cause a marked decrease in the rate of degradation of ER-retained proteins [6].

The UPS has been implicated in the degradation of proteins from other organelles, most notably the ER. The ER has garnered the most attention because it requires active protein quality control. A large amount of misfolded or unassembled membrane, secretory proteins as well as ER 
luminal proteins are degraded by the ERassociated degradation (ERAD) $[7,8]$.

Despite ERAD was originally described as quality control system for misfolded proteins, it has wide functions as a protein degradation system [9]. ERAD is conserved in all eukaryote systems and consists of a complex set of E1, E2, E3 processes that are responsible for ubiquitylating and degrading proteins. In budding yeast, ERAD consists of only one E1 enzyme, named Uba1, which is essential for viability [10]. By contrast, it has been found that 11 different E2 enzymes (Ubc1-13) but only three are part of ERAD, Ubc1, Ubc6 and Ubc7 [11]. Concerning E3s there are two well documented, Hrd1/Der3 (which mainly interacts with Ubc7 but also weakly with Ubc1 [12]) and Doa10 (which interacts with Ubc6 and Ubc7). These E3s recognize the specific sequences (commonly known as degron) that initially impact the turnover of the proteins in the cytoplasmic domains (ERAD-C), luminal domain (ERAD-L) o membrane bound ERproteins (ERAD-M) which are the substrates [13]. The E3 ubiquitin ligase $\mathrm{Hrd1}$, assisted by another subunit named Der1, is involved in targeting proteins located at the lumen (ERAD-L). Hrd interacts mostly with Ubc7 which is recruited to the ER and activated through Cue1. The Cdc48 which is an AAA ATPase is recruited to the ER by the adaptor protein Ubx2. Then, ubiquitylated substrates are cleared out from the ER by Cdc48 and cofactors Ufd 1 and Npl4 [9]. The other E3 ubiquitin ligase, Doa10, acts on membrane (ERAD-M) or cytosolic (ERAD-C) ER proteins. In this case, $\mathrm{Cdc} 48$ is only required for ERAD-M but not for ERAD-C [9].

Rot1 is an essential protein in the budding yeast Saccharomyces cerevisiae, which was identified in a search for second-site suppressors of a tor2 thermosensitive mutation [14]. Tor2 is involved in growth control and actin cytoskeleton organization. We previously demonstrated that Rot1 protein is involved in several cellular functions as protein folding and cell wall biosynthesis [15, 16], actin cytoskeleton dynamics and cell cycle control [17]. Rot1 is primarily located at the endoplasmic reticulum-nuclear membrane where it is translocated through two internal topogenic elements by an SRP-independent posttranslational mechanism which depends on Sec62 [18, 19]. Rot1 is a membrane protein with unusual topology because the $\mathrm{N}$-terminal of the protein faces the ER lumen, where it is glycosylated, and the single C-terminal hydrophobic region, which contains an essential serine residue at position 250, is essential for proper protein function and cell viability [18-20]. We also found that Rot1 protein genetically interacts with the proteasome [17]. Despite Rot1 cellular levels are critical for yeast survival, how Rot1 gets degraded in cells has not been investigated.

To get deeper insights about how Rot1 protein levels are controlled in cells, we used mutant cells components of the cell degradation system and monitored Rot1 protein levels in shut-off assays. Here we show that Rot1 turnover requires the ubiquitin conjugating E2, Ubc6 and $\mathrm{Ubc7}$, and one of the E3 ubiquitin ligases, Hrd1 but not Doa1 from the ER-associated degradation system (ERAD), and ultimately the pre1 pre2 subunits of the ubiquitin proteasome system (UPS).

\section{Results and Discussion}

The ubiquitin proteasome system plays an essential role in Rot1 protein levels. The fact that Rot1 genetically interacts with the proteasome [17, 18] prompted us to investigate whether the UPS could be involved in Rot1 protein degradation. First, Rot1 levels was assayed in promoter shutoff experiments, in which the expression of a GAL1:ROT1-HA gene was repressed by adding glucose to the medium in WT cells and the rate of Rot1 degradation was 
Juanes MA et al

determined by HA-epitope tagged ROT1 western analysis. Using this approach, we observed that Rot1 was a short-lived protein. In wild-type cells Rot1 was almost undetectable within 45-60 min after the repression of the GAL promoter (Figure 1). In parallel, same assays were performed using cell mutants in the pre 1 pre 2 subunits (components of the $20 \mathrm{~S}$ which impact on the structure of the active site of the proteasome) and transformed with a plasmid containing the gene GAL1:ROT1$H A$. In contrast to WT cells, Rot1 protein levels poorly decay in the pre1 pre2 cells bearing the GAL1:ROT1-HA plasmid, persisting for more than 120 min after repression of the GAL promoter (Figure 1). This result confirmed that Rot1 is degraded through the ubiquitin proteasome system.

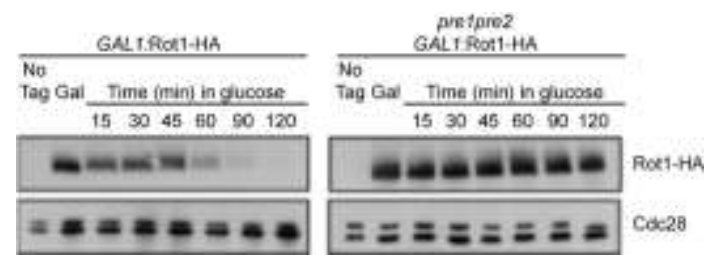

Figure 1. Analysis of the Rot1 protein levels in the mutant strain in the proteasome subunits pre1pre2. Cells of wild type (WCG4 $\alpha$ ) and mutant pre1 pre2 (WCG4$11 / 22$ ) transformed with a plasmid that contains the Rot1-HA protein under GAL1 promoter were grown in SD Gal overnight and then transferred to glucose medium. The levels of the Rot 1 protein Rot1 after the repression of the GAL1 promoter by glucose were analyzed by Western blot at the indicated times. A cell extract obtained from the wild strain (W303) is included as negative control (no tag). The level of protein Cdc28 is shown as loading control.

Similar results were obtained when expressing GAL-ROT1-HA endogenous or ectopically using a plasmid we previously generated [18], indicating that expressing of Rot1 does not change its stability in cells (data not shown). Same decay kinetics was also obtained when performing translational shut-off experiments carried out with the same cells to follow Rot1 decay after addition of cycloheximide by western blot (data not shown).

Turnover of Rot1 proceeds via the two E2 proteins from ERAD, Ubc6 and Ubc7 Rot 1 is a transmembrane protein primarily located at the endoplasmic reticulum (ER) facing the lumen [18]. Since the prevalent pathway for ER-membrane protein turnover is ERAD, we hypothesized that ERAD could mediate Rot1 degradation. We performed shut-off experiments in the three documented ubiquitin conjugating enzymes (E2s) yeast mutant cells (Ubc1, Ubc6 and Ubc7) components of the ERAD pathway [2, 9] while expressing the plasmid containing the gene GAL1:ROT1-HA.

Because $U B C 1$ gene is essential for viability, we used a conditional mutant strain that expresses the $U B C 1$ gene under the control of the doxycycline-regulated tetO7 promoter (tet $O_{7}: U B C 1$ ). In cells growing in the presence of $10 \mu \mathrm{g} / \mathrm{mL}$ doxycycline, i.e. deficient in UBC1, and after GAL1:ROT1-HA repression by glucose to the culture cells, we observed that the rate of Rot1 degradation was indistinguishable from control cells. This suggested that Rot1 degradation is not Ubc1-dependent (Figure 2). Next, we studied the other two E2 enzymes involved in ERAD: Ubc6 and Ubc7. We compared Rot1 degradation in $4 b c 6$ and ubc7 cells versus WT cells. Both mutants show similar Rot1 levels, being detectable by western blot throughout the two-hour experiment (Figure 3 ). These results suggest that Ubc6 and Ubc7 are regulating Rot1 protein 
4

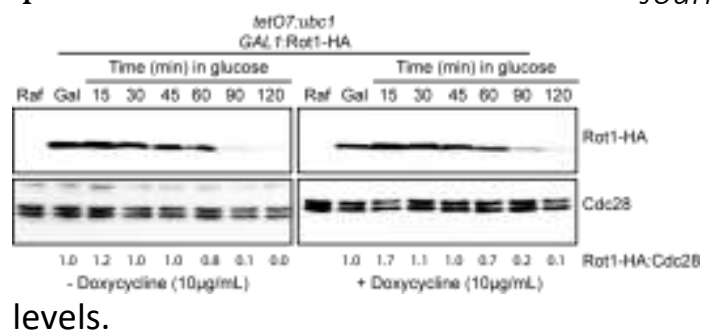

Figure 2. Analysis of the stability of the protein Rot1 in the mutant strain in the E2 enzyme Ubc1. Cultures of the mutant strain tet07: ubc1 transformed with a plasmid containing the Rot1-HA protein under the GAL1 promoter were grown in the presence or absence of doxycycline $(10 \mu \mathrm{g} / \mathrm{mL})$ and in raffinose (Raf) as a carbon source. Then galactose was added to the medium (Gal) during $1 \mathrm{~h}$ and cells were transferred to medium with glucose. The levels of the Rot1 protein after repression of the GAL1 promoter were analyzed by Western blot at the indicated times. The level of protein Cdc28 is shown as loading control. Below each lane of the blot is the ratio of Rot 1 to Cdc28 relative to the first time point in Gal ( $\mathrm{t}=0 \mathrm{~min})$.

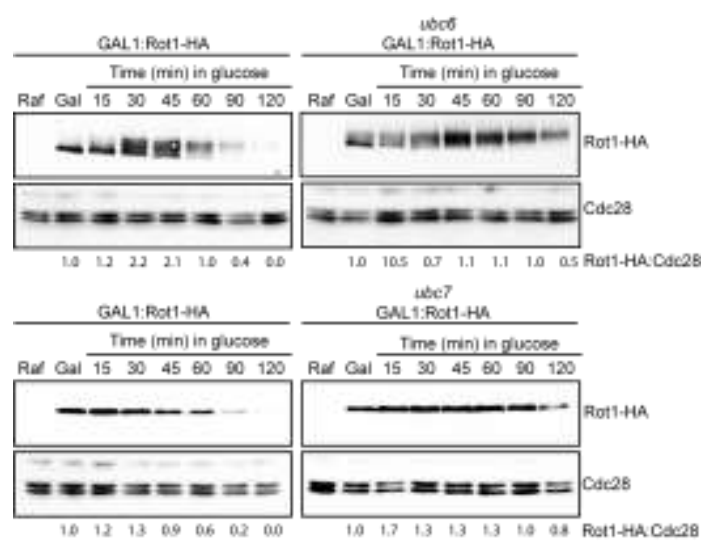

Figure 3. Analysis of the stability of the protein Rot1 in the mutant strains in the enzymes E2: Ubc6 and Ubc7. Cultures of the yeast mutant strains $u b c 6$ and $u b c 7$ transformed with a plasmid containing the Rot1-HA protein under the GAL1promoter were grown in raffinose (Raf) as a carbon source. Then galactose was added to the medium (Gal) during $1 \mathrm{~h}$ and cells were transferred to medium with glucose. The
Postdoctoral Research November 2017: 3-10 levels of the Rot1 protein were analyzed by Western blot at the indicated times. The level of the $\mathrm{Cdc} 28$ protein is shown as loading control. Below each lane of the blot is the ratio of Rot 1 to $\mathrm{Cdc} 28$ relative to the first time point in $\mathrm{Gal}(\mathrm{t}=0 \mathrm{~min})$.

We wondered if the two E2 activities could be synergizing Rot1 degradation in cells. To test this, we performed similar shut off experiments using the $u b c 6 u b c 7$ double mutant. As expected in WT cells, Rot1 levels are basically undetectable after 45-60 min of GAL1 promoter repression (Figure 4). In sharp contrast, ubc6 ubc7 mutant cells Rot1 protein levels barely decay during the 2 hours of the experiment (Figure 4).

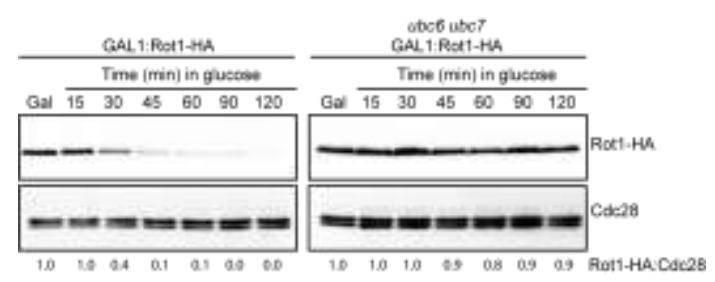

Figure 4. Analysis of the stability of the protein Rot1 in the double mutant in the enzymes E2: Ubc6 / Ubc7. Cultures of the yeast mutant strains $u b c 6 u b c 7$ transformed with a plasmid containing the Rot1-HA protein under the GAL1 promoter were grown in raffinose (Raf) as a carbon source. Then galactose was added to the medium (Gal) during $1 \mathrm{~h}$ and cells were transferred to medium with glucose. The levels of the Rot1 protein were analyzed by Western blot at the indicated times. The level of the Cdc28 protein is shown as loading control. Below each lane of the blot is the ratio of Rot1 to Cdc28 relative to the first time point in Gal ( $\mathrm{t}=0 \mathrm{~min})$.

We noticed that the Rot1's protein pattern was slightly different in $u b c 6$ than in $u b c 7$ cells. It presented some smearing and diffuse band pattern in absence of UBC6. Then, these results show that Rot1 turnover is mediated by both E2, Ubc6 and/or Ubc7 and these enzymes may teamwork sequentially to achieve proper Rot1 
Juanes MA et al

degradation. It could be plausible that Rot1 gets mono-ubiquitylated by Ubc6 and triggers Rot1 ubiquitylation by $\mathrm{Ubc} 7$ to enhance Rot1 degradation.

The ERAD E3-ubiquitin ligase Hrd1 plays a role in Rot1 degradation. In budding yeast, there are at least two well-known E3ubiquitin ligase associated to the ERAD pathway, Hrd1/Der3 and Doa10 [9]. Following same strategy than for the E2 study, we checked Rot1 degradation in cells lacking those E3-ubiquitin ligase. We monitored Rot1 levels in the hrd1 doa10 double mutant (Figure 5). Notably, Rot1 levels were stabilized yet highly diffused in the E3-ubiquitin ligase double mutant cells, a pattern never detected in WT cells. The shift observed in Rot1 mobility could be attributed to Rot1 other posttranslational modifications such as glycosylation or phosphorylation.
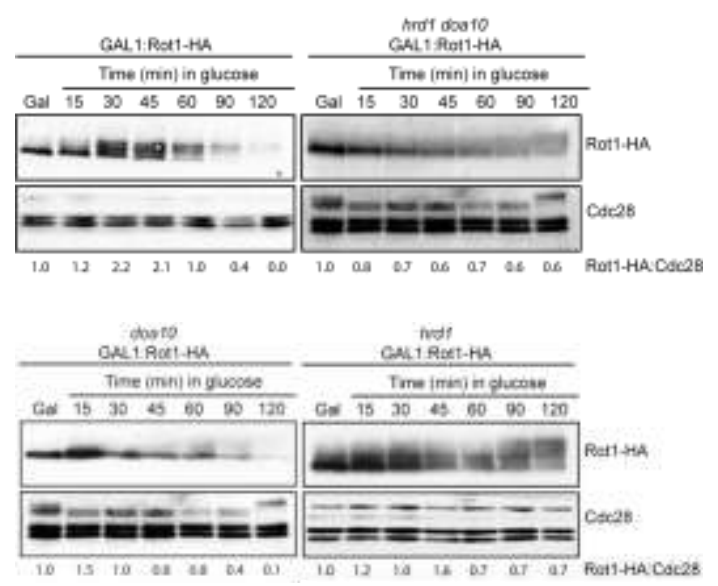

Figure 5. Analysis of the stability of the protein Rot1 in the double mutant in the enzymes E3: Hrd1 / Doa10 and the individual mutant strains Hrd1 and Doa10. Cultures of the yeast mutant strains hrd1doa10 transformed with a plasmid containing the Rot1-HA protein under the GAL1promoter were grown in raffinose (Raf) as a carbon source. Then galactose was added to the medium (Gal) during $1 \mathrm{~h}$ and cells were transferred to medium with glucose. The levels of the Rot1 protein were analyzed by Western blot at the indicated times. The level of the $\mathrm{Cdc} 28$ protein is shown as loading control. Below each lane of the blot is the ratio of Rot1 to Cdc28 relative to the first time point in Gal $(\mathrm{t}=0$ $\min$ ).

In order to determine whether one or both E3 contributes to Rot1 degradation, same experimental approach was performed but using the $h r d 1$ or doal individual mutant cells. The results obtained suggest that Hrd1 could be the only E3-ubiquitin ligase promoting Rot1 degradation because we could not observe any appreciable change in Rot1 degradation rate in the doa1 mutant cells versus WT cells (Figure 5 ). Moreover, hrd1 mutant cells showed a shift in Rot1 levels similar than that observed in the hrd1 doa10 double mutant, pointing that the protein gets ubiquitylated by not properly degraded in absence of $\mathrm{Hrd1}$.

Then, a possible scenario could be that Rot 1 is degraded by ERAD (Figure 6). On one side, the chief ubiquitin-conjugating enzymes E2, Ubc6 and Ubc7, are required for Rot1 degradation either in a cooperative or redundant manner. The resulting ubiquitin-conjugating E2-Rot1 complex is then processed at the ER by the E3ubiquitin ligase Hrd1 but not by Doa1. Interestingly, $\mathrm{Hrd} 1$ is responsible of degrading misfolded intramembrane proteins (ERAD-M) or proteins that localize in the lumen (ERAD-L), by contrast to Doa1 that targets misfolded cytosolic domain of proteins around the ER (ERAD-C) [21]. Then, this agrees with Hrd1 targeting Rot1, which faces the lumen of the $E R$, for degradation. However, we believe that during the course of our experiments Rot1 protein is kept in its native form and not misfolded. Thus, these results claim the question whether Hrd1 is capable to degrade a broader spectrum of proteins that has been assumed so far. In short, our findings reveal that Rot1 is a protein with a short half-life 
and is degraded via ERAD but also open a new avenue for further investigations about the plethora of substrates that Hrd1 E3ubiquitin ligase targets in cells.

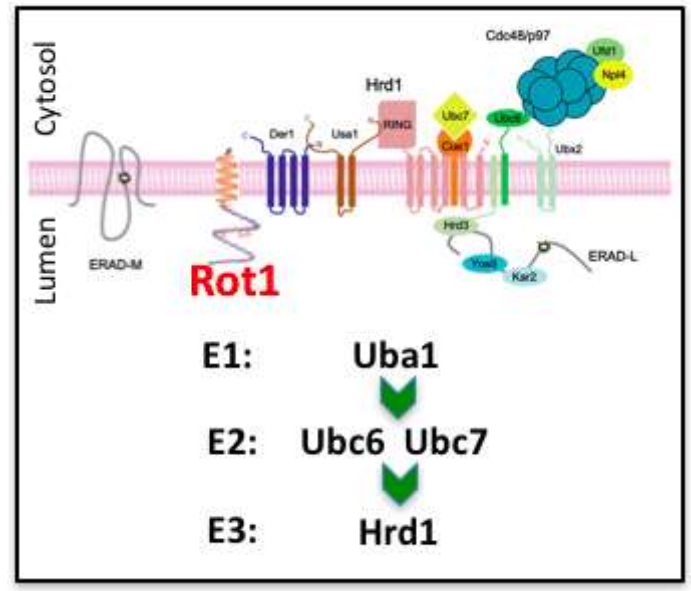

Figure 6. Schematic of Rot1 domains and ERAD machinery located at the ER. Below the schematic of the E1, E2, E3 identified as required for Rot1 degradation.

\section{Materials and methods}

Yeast Strains, Genetic Methods, and Plasmids. All ERAD yeast strains were derivatives of W303 (ade2-1, trp1-1, leu23,112, his3-11,15, ura3, ssd1) kindly provided by $M$. Hochstrasser. Wild-type (WCG4 $\alpha$ ) and mutant pre1pre2 (WCG411/22) obtained generously from S.D. Wolf. The strain tet0 $7: U B C 1$ gene was acquired from Open Biosystems. Cells were grown on standard yeast synthetic dextrose (SD), or rafinose (SRaf) or galactose (SGal) medium supplemented as required. Doxycycline (Sigma) was added at a concentration of $10 \mu \mathrm{g} / \mathrm{mL}$ to fully repress the tetO $_{7}$ promoter. Yeast strain GAL1:ROT1-HA in W303 background and plasmid pGAL:ROT1-HA in which the ROT1 promoter was substituted for the GAL1 promoter either in the genome or in the plasmid pROT1-HA-CEN (centromeric and encoding three copies of the HA epitope integrated in frame in the middle region of the protein) have been previously described in [18].
Rot1 protein stability assays. To evaluate the Rot1 protein decay, WT strains and the different mutant cell studied carrying the pGAL1:ROT1-HA plasmid were grown directly on galactose synthetic medium (SGal) or in rafinose (SRaf). Then, to repress the GAL1 promoter, glucose to a final concentration of $2 \%$ was added to the media. Cells were collected at different times during $120 \mathrm{~min}$ and processed for Western blot analysis. To evaluate the Rot1 protein decay in ubc1 mutant cells (tet $0_{7}: U B C 1$ gene), cells were incubated in the presence of 10 ag/mL doxycicline for at least 6 hours to shut off UBC1 gene expression and then glucose was added to repress GAL1 promoter as above indicated.

Western blot analysis. Approximately $10^{8}$ cells were collected, resuspended in $100 \mu \mathrm{L}$ of water, and after adding $100 \mu \mathrm{L}$ of $0.2 \mathrm{M}$ $\mathrm{NaOH}$, they were incubated for 10 min at room temperature. Cells were collected by centrifugation, resuspended in $50 \mu \mathrm{L}$ of sample buffer (150 mM Tris- $\mathrm{HCl}(\mathrm{pH} 6.8)$, 300 mM DTT, 6\% SDS, 0.3\% bromophenol blue, and $30 \%$ glycerol) and incubated for 5 $\min$ at $95 \circ$ C. Extracts were clarified by centrifugation, and equivalent amounts of protein were resolved by SDS-PAGE. After transfer to nitrocellulose filters, tagged proteins were detected with the corresponding antibody (anti HA 3F10 rat monoclonal antibody from Roche, dilution 1:1000) for western detection of Rot1-HA, anti-PSTAIRE antibody (Santa Cruz) for western detection of $\mathrm{Cdc} 28$, dilution 1:1000) and the ECL Advance Western blotting detection Kit (GE Healthcare) following the manufacturer's instructions

\section{Acknowledgments}

We are very grateful to Dr. Hochstrasser and Dr. D.H. Wolf for kindly supplying yeast strains. This work was supported by the Spanish Government and co-financed by ERDF from the European Union (grants number BFU2013-47503 and BFU2014- 
Juanes MA et al

58429-P) and by the Generalitat Valenciana (grant number GVPROMETEO2016-123). M.A.J. was supported by a Generalitat Valenciana Fellowship.

\section{References}

1. Mayor T, Sharon M, Glickman MH. Tuning the proteasome to brighten the end of the journey. Am J Physiol, Cell Physiol. 2016;311:C793-804. https://doi.org/10.1152/ajpcell.00198.2016 PMid:27605452

2. Hochstrasser M. Ubiquitin-dependent protein degradation. Annu Rev Genet. 1996;30:405-39. https://doi.org/10.1146/annurev.genet.30.1.405 PMid:8982460

3. Leggett DS, Glickman $M H$, Finley $D$. Purification of proteasomes, proteasome subcomplexes, and proteasome-associated proteins from budding yeast. Methods Mol Biol. 2005;301:57-70.

https://doi.org/10.1385/1-59259-895-1:057

4. Budenholzer L, Cheng CL, Li Y, Hochstrasser $\mathrm{M}$. Proteasome structure and assembly. J Mol Biol. 2017.

https://doi.org/10.1016/j.jmb.2017.05.027

PMid:28583440

5. Seufert $W$, Jentsch S. In vivo function of the proteasome in the ubiquitin pathway. EMBO J. 1992;11:3077-80.

PMid:1322295

PMCid:PMC556791

6. Biederer $\mathrm{T}$, Volkwein $\mathrm{C}$, Sommer $\mathrm{T}$. Degradation of subunits of the Sec61p complex, an integral component of the ER membrane, by the ubiquitin-proteasome pathway. EMBO J. 1996;15:2069-76.

PMid:8641272

PMCid:PMC450128

7. Bonifacino J. UBIQUITIN AND THE CONTROL OF PROTEIN FATE IN THE SECRETORY AND ENDOCYTIC PATHWAYS1.

8. Thibault G, Ng DTW. The endoplasmic reticulum-associated degradation pathways of budding yeast. Cold Spring Harb Perspect Biol. 2012;4.

https://doi.org/10.1101/cshperspect.a013193

9. Zattas D, Hochstrasser M. Ubiquitindependent protein degradation at the yeast endoplasmic reticulum and nuclear envelope. Crit Rev Biochem Mol Biol. 2015;50:1-17. https://doi.org/10.3109/10409238.2014.959889 PMid:25231236 PMCid:PMC4359062

10. McGrath JP, Jentsch S, Varshavsky A. UBA 1: an essential yeast gene encoding ubiquitinactivating enzyme. EMBO J. 1991;10:227-36. PMid:1989885 PMCid:PMC452634

11. Lemus L, Goder V. Regulation of Endoplasmic Reticulum-Associated Protein Degradation (ERAD) by Ubiquitin. Cells. 2014;3:824-47.

https://doi.org/10.3390/cells3030824

PMid:25100021 PMCid:PMC4197631

12. Bays NW, Gardner RG, Seelig LP, Joazeiro CA, Hampton RY. Hrd1p/Der3p is a membraneanchored ubiquitin ligase required for ERassociated degradation. Nat Cell Biol. 2001;3:24-9. https://doi.org/10.1038/35050524 PMid:11146622

13. Kawaguchi S, Ng DTW. SnapShot: ERassociated protein degradation pathways. Cell. 2007;129:1230.

https://doi.org/10.1016/i.cell.2007.06.005

PMid:17574032

14. Bickle M, Delley PA, Schmidt A, Hall MN. Cell wall integrity modulates RHO1 activity via the exchange factor ROM2. EMBO J. 1998;17:223545.

https://doi.org/10.1093/emboj/17.8.2235

PMid:9545237 PMCid:PMC1170568

15. Machi K, Azuma M, Igarashi K, Matsumoto T, Fukuda $H$, Kondo $A$, et al. Rot1p of Saccharomyces cerevisiae is a putative membrane protein required for normal levels of the cell wall 1,6-beta-glucan. Microbiology (Reading, Engl). 2004;150 Pt 10:3163-73. https://doi.org/10.1099/mic.0.27292-0

PMid:15470097

16. Takeuchi $M$, Kimata $Y$, Hirata A, Oka M, Kohno K. Saccharomyces cerevisiae Rot1p is an ER-localized membrane protein that may function with $\mathrm{BiP} / \mathrm{Kar} 2 \mathrm{p}$ in protein folding. J Biochem. 2006;139:597-605. https://doi.org/10.1093/jb/mvj063

PMid:16567426 
17. Juanes MA, Queralt E, Ba-ó MC, Igual JC. Rot1 plays an antagonistic role to $\mathrm{Clb} 2$ in actin cytoskeleton dynamics throughout the cell cycle. J Cell Sci. 2007;120 Pt 14:2390-401. https://doi.org/10.1242/ics.002758

PMid:17606994

18. Juanes MA, Carlos Igual J, Carmen Ba-ó M. Membrane topology and post-translational modification of the Saccharomyces cerevisiae essential protein Rot1. Yeast. 2008;25:93-106. https://doi.org/10.1002/yea.1549

PMid:17914748

19. Juanes MA, Martínez-Garay CA, Igual JC, Baó MC. Targeting and membrane insertion into the endoplasmic reticulum membrane of Saccharomyces cerevisiae essential protein Rot1. FEMS Yeast Res. 2010;10:639-47. https://doi.org/10.1111/i.1567- 1364.2010.00653.x PMid:20608986

20. Martínez-Garay CA, Juanes MA, Igual JC, Mingarro I, Ba-ó MC. A transmembrane serine residue in the Rot1 protein is essential for yeast cell viability. Biochem J. 2014;458:239-49. https://doi.org/10.1042/BJ20131306

PMid:24303792

21. Carvalho P, Goder V, Rapoport TA. Distinct ubiquitin-ligase complexes define convergent pathways for the degradation of ER proteins. Cell. 2006;126:361-73. https://doi.org/10.1016/j.cell.2006.05.043

PMid:16873066 